\title{
Protective effect of Luteolin on atopic dermatitis murine model via IgE mediated immune response
}

\author{
Jian Wang ${ }^{a}$, Gang Li ${ }^{b}$, Haixiu Zhang ${ }^{c}$ and Ying Xie ${ }^{b}$ \\ ${ }^{a}$ Department of Traditional Chinese Medicine, The Second People Hospital of Dezhou, Dezhou, Shandong Province, 253000, China; \\ ${ }^{b}$ Department of Pharmacy, Central Hospital Affiliated to Shandong First Medical University, Jinan, Shandong Province, 250013, \\ China; 'Department of Dermatology, Jinan Municipal Hospital Of Traditional Chinese Medicine, Jinan, Shandong, 250012, China
}

\begin{abstract}
Atopic dermatitis (AD) is a serious inflammatory condition associated with severe itching and persistent eczematous lesion. Therefore, the present study was intended to scrutinize the beneficial effect of Luteolin (LT) on the atopic dermatitis murine model. The effect of LT was investigated on the various parameters, such as oxidative stress and inflammation after induction of AD. The serum level of IgE, and cells of the WBC family (neutrophils, basophils, eosinophils, monocytes, lymphocytes, and total WBC) and histopathological analysis of skin tissue were also examined to confirm the effect of LT. Results of the study suggested that LT significantly inhibited the elevated IgE level together with improvement in injured skin tissue architecture. It also reduces oxidative stress (MDA, SOD, and GSH) and inflammation (IL-1 $\beta$, IL-6, TNF- $\alpha$, IFN- $\gamma$, IL-4, and IL-17A) as evidenced by ELISA analysis. The level of examined WBC family cells was found reduced significantly as compared to the AD model group. In western blot analysis, LT showed significant down-regulation of NF-KB and TLR-4. Collectively, our results suggest that LT can effectively reverse the effect of atopic dermatitis via improving immunological response.
\end{abstract}

\section{ARTICLE HISTORY}

Received 25 November 2020 Accepted 23 February 2021

\section{KEYWORDS}

atopic dermatitis; inflammation; oxidative stress; IgE, NF-kB

\section{Introduction}

Atopic dermatitis (AD) is a serious inflammatory condition associated with severe itching and persistent eczematous lesion. According to an estimate, it affects nearly $10-20 \%$ of children and $5 \%$ of adults worldwide (Galli et al. 2020; Langan et al. 2020). The correct etiology of $A D$ is still unknown, however, many studies reported the close connection between environmental and genetic factors that leads to impairment of skin barrier and immune system (Leung et al. 2004). The clinical management of $A D$ is heavily dependent upon the use of glucocorticoids either systemic or locally. However, the long-term use of these steroidal drugs is often associated with serious complications that limit their usefulness (Emanuel and Hawarden 2018). Thus, newer agents or therapies are needed to treat $A D$ in a much efficient way with fewer side-effect.

Natural products have a long history in traditional Chinese medicine (TCM) in various forms of herbal preparations. They have been used all over the world for hundreds or even thousands of years, and now have blossomed into orderly-regulated systems of medicine (Arikawa et al. 2002; Thomsen 2014; Marsella and De Benedetto 2017). The uniqueness of the natural product lies in their chemical, which results in diversity in their biological activities and drug-like properties. Luteolin (LT), belongs to the chemical classification of flavonoids obtained from perilla, parsley, and green peppers (Lin et al. 2008). It has a very strong reactive oxygen species (ROS) scavenging property which renders it useful against several diseases such as cancer, inflammatory conditions, microbes, and diabetes. Recently, it showed excellent pharmacological activity against canine atopic dermatitis (Gugliandolo et al. 2020), but no study has till now explored the potential benefit of LT against human AD (Seelinger et al. 2008). Thus, in the present study, we wish to scrutinize the effectiveness of $L T$ against humans like $A D$ in the murine model.

CONTACT Ying Xie xieyingjn001@sina.com 


\section{Experimental}

\section{Animals}

Eight-week-old male BALB/c mice (weight 20-22 g) were housed under specific-pathogen-free (SPF) conditions. All animals were treated following the Institutional Animal Care and Use Committee (IACUC) and the study protocols were approved by the Committee on Animal Research and Care of SFMU.

\section{DNCB-induced dermatitis animal model}

The dermatitis animal model induced by DNCB was adapted from the previous study (Wu et al. 2019). Briefly, $150 \mu \mathrm{L} /$ mouse of $2 \%$ DNCB solution (dissolved in a 3:1 mixture of acetone and olive oil) was applied on approximately $4 \mathrm{~cm}^{2}$ of dorsal skin on each BALB/c mouse after hair removal. Mice were then housed without any further treatment for the next four days followed by the application of $150 \mu \mathrm{L} /$ mouse of $0.2 \%$ DNCB on the dorsal skin every three days throughout the experiment.

\section{Luteolin (LT) treatment}

LT was purchased directly from Sigma-Aldrich Co. (St. Louis, MO) and dissolved in the corn oil/DMSO vehicles $(v / v, 95 / 5)$ before use. Three-treated groups $(n=6$ for each group) were given 10,20 , and $30 \mathrm{mg} / \mathrm{kg}$ of LT via intraperitoneal injection every other day from day 7 to day 31 over the animal experiment. Two control cohorts were incorporated in this study: normal control (sham) which was exposed to a mixture of acetone and olive oil without treatment with no DNCB sensitization, and vehicle control (AD model), defined as DNCB-sensitized and injected with oil/DMSO vehicles with no LT administration.

\section{Measurement of serum IgE levels}

Cardiac blood samples were collected from the mice after euthanasia by asphyxiation with $\mathrm{CO}_{2}$. Serum samples were obtained after centrifugation $(10,000 \times \mathrm{g}$, $10 \mathrm{~min}$ ) and stored at $80^{\circ} \mathrm{C}$ until quantitative analysis. Serum total IgE levels were determined by sandwich ELISA using the BD PharMingen ELISA set according to the manufacturer's instruction (San Jose, CA).

\section{Analysis of mouse blood}

Whole blood samples were collected by murine cardiac puncture upon euthanizing. The blood was placed in Vacutainer TM tubes containing EDTA (BD Science, Franklin Lakes, NJ). Anti-coagulated blood samples were submitted for determination of hematological parameters (white blood cells (WBCs), lymphocytes, monocytes, eosinophils, basophils, and neutrophils) in a HEMAVET 950 hematology analyzer (Drew Scientific, Inc., Miami Lakes, FL) following the manufacturer's recommendation.

\section{Evaluation of MDA, GSH, and SOD}

Blood samples were allowed to clot for $2-3 \mathrm{~h}$, and serum was separated by centrifugation at $2 ; 200 \times g$ for $10 \mathrm{~min}$ and stored at $4{ }^{\circ} \mathrm{C}$ for the estimation of the level of MDA, GSH, and SOD using commercially available ELISA (Jiangsu Enzyme Free Experimental Co., Ltd. China) kits as per the manufacturer protocol.

\section{Evaluation of pro-inflammatory cytokines}

The serum level of IL-1 $\beta$, IL-6, TNF- $\alpha$, IFN- $\gamma$, IL-4, and IL$17 \mathrm{~A}$ were determined by solid-phase sandwich ELISA kits as per the manufacturer protocol (Jiangsu Enzyme Free Experimental Co., Ltd. China) $1.43 \mathrm{pg} / \mathrm{ml}(\mathrm{IL}-1 \beta)$, $2.55 \mathrm{pg} / \mathrm{ml}$ (IL-6), $0.48 \mathrm{pg} / \mathrm{ml}$ (IL-4), $3.3 \mathrm{pg} / \mathrm{ml}$ (IL-17A), $7.14 \mathrm{pg} / \mathrm{ml}$ (TNF-a), $11.15 \mathrm{pg} / \mathrm{ml}$ (IFN- $\gamma$ ). The minimum detectable dose was determined by adding two standard deviations to the mean optical density value of ten zero standard replicates and calculating the corresponding concentration. Serum samples were diluted 1:5 or 1:10 in PBS. Concentrations were reported as pg/ $\mathrm{ml}$. All analyses and calibrations were performed in duplicate. Optical densities were determined using an absorbance microplate reader (Elx808 ${ }^{\mathrm{TM}}$; Bio-Tek Instruments, Winooski, VT, USA) at $450 \mathrm{~nm}$. GraphPad Prism Data Analysis software 6 (GraphPad Software, Inc., La Jolla, CA, USA). was used to analyze all materials and depict the standard curve.

\section{Western blotting}

The skin tissues were lysed with RIPA buffer, PMSF, and phosphorylase inhibitor cocktail (MCE, New Jersey, USA) mixed according to 100:1:1. The BCA protein quantitation kit (Boster, California, USA) was used to determine the protein concentration. Then the loading buffer was added to the protein sample and boiled for $10 \mathrm{~min}$ at $100^{\circ} \mathrm{C}$ to denature the proteins. The protein extract $(30 \mu \mathrm{g})$ was resolved by $12 \%$ SDS-PAGE electrophoresis and electrotransferred onto a membrane of 
nitrocellulose. The membrane was blocked-up using TrisPBS (TPBS) with Tween 20 and $5 \%$ skimmed milk and probed with primary antibodies: Next, the secondary antibody conjugated to HRP (1:10000; Abcam/Cell Signaling Technology) in TPBS was added and incubated at $37^{\circ} \mathrm{C}$ for $1 \mathrm{~h}$. Bands were visualized using an enhanced chemiluminescence image analyzer, and protein levels were quantified using Image-Pro Plus software (Media Cybernetics, Inc., MD, USA).

\section{Statistical analysis}

The data were articulated as mean \pm standard error (SEM). The statistical analysis was performed using one-way analysis of variance (ANOVA) followed by Bonferroni post hoc multiple comparison test (GraphPad Prism 5.0, USA). The P-value $<0.05$ was considered statistically significant.

\section{Results}

\section{Effect LT on the IgE level of skin histopathology}

The effect of $L T$ was first investigated on the expression of $\mathrm{IgE}$ in a murine model. As shown in Fig. $1 \mathrm{~A}$, the level of IgE was found highly elevated in the disease model group, as compared to the control. However, upon administration of $L T$, the elevated level of IgE was found significantly reduced. Moreover, upon observing the histopathological changes as shown in Fig $1 B$, the AD group showed significantly increased epidermal hyperplasia along with infiltration of inflammatory cells as compared to control. However, groups treated with LT showed restoration of an abnormal architecture of the skin near to normal as compared to the disease model with decreased infiltration of inflammatory cells.

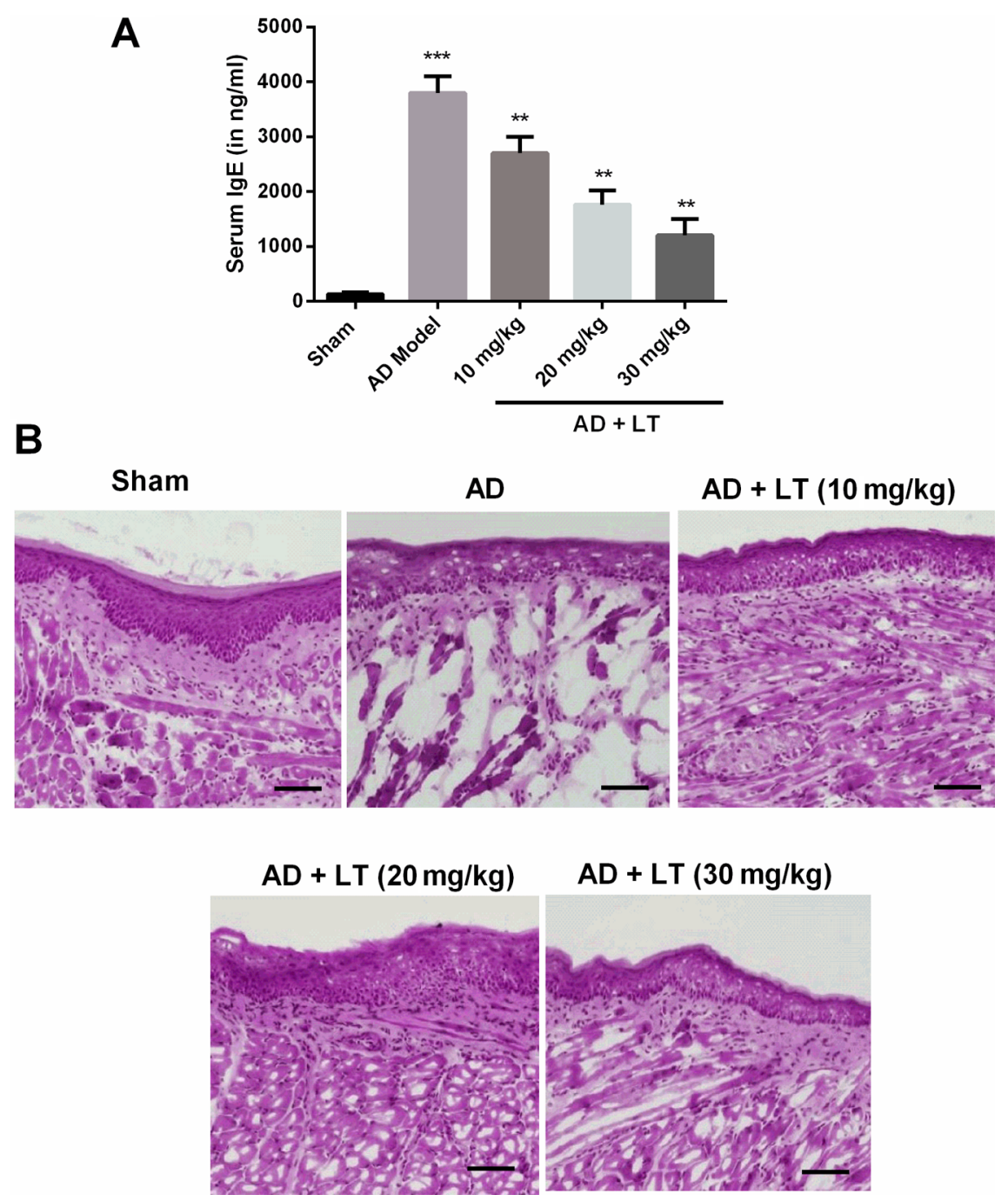

Figure 1. Effect of $L T$ on $(A)$ serum IgE level and (B) histopathology of tissues. Values represent the mean $\pm S E M .{ }^{* * *} P<0.05$ vs control; ${ }^{* *} P<0.05$ vs $A D$, one-way analysis of variance (ANOVA) followed by Bonferroni post hoc test. 


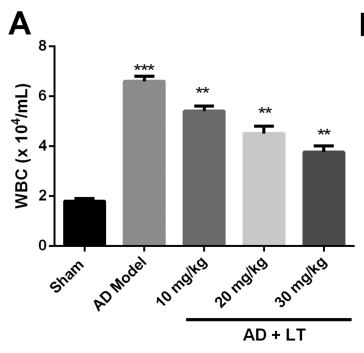

B

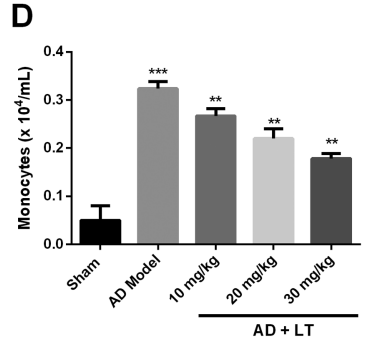

E
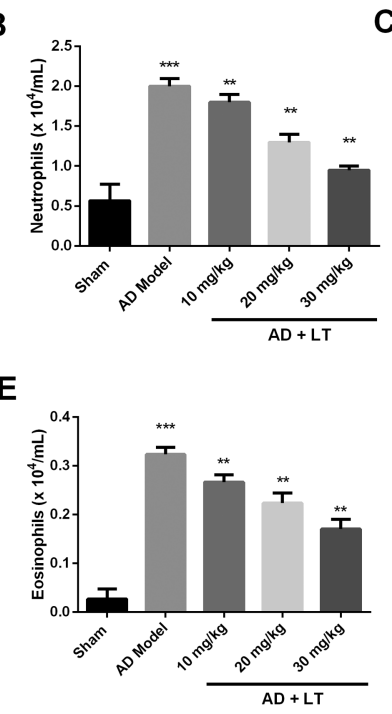

C

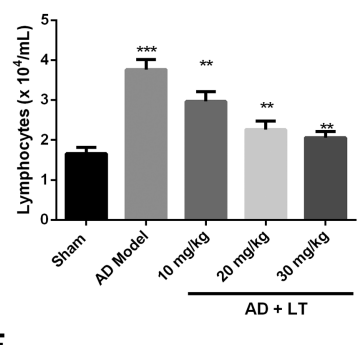

$\mathbf{F}$

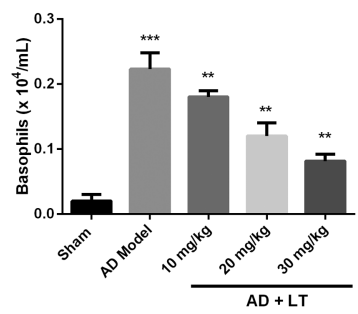

Figure 2. Effect of LT on WBC cell population, where (A) WBC, (B) Neutrophils, (C) Lymphocytes, (D) Monocytes, (E) Eosinophils, and (F) Basophils. Values represent the mean \pm SEM. ${ }^{* * *} P<0.05$ vs control; ${ }^{* *} P<0.05$ vs $A D$, one-way analysis of variance (ANOVA) followed by Bonferroni post hoc test.

\section{Effect of LT on the WBC family cells}

To understand the anti-inflammatory effect of LT, next, we have examined the level of WBC family cells, such as eosinophils in murine blood samples after establishing AD. As shown in Fig. 2, the level of examined cells (neutrophils, basophils, eosinophils, monocytes, lymphocytes, and total WBC) was found highly elevated in the AD model as compared to control. In LT treated group, the level of these tested cells was significantly restored to near to normal in comparison to the $A D$ group.

\section{Effect on the pro-inflammatory cytokines}

Based on the above observation where LT significantly decreased the level of various blood cells, next, we thought to investigate whether LT has any inhibitory effect on the pro-inflammatory cytokines. The effect of LT was investigated on the level of IL-1 $\beta$, IL- 6 , TNF- $\alpha$, IFN- $\gamma$, IL-4, and IL-17A. As shown in Fig. 3, the level of these tested cytokines was found highly increased in the AD model group as a control. Upon introduction of LT, the level of these cytokines was reduced significantly in a dose-dependent manner.

\section{Effect of LT on the oxidative stress indices}

The effect of $L T$ was also investigated on the various indices of oxidative stress as shown in Fig. 4. It has been found that the disease model group showed an elevated level of MDA with a reduced level of SOD and GSH as compared to sham. On the contrary, as compared to the disease model, the LT treated group, the level of SOD and GSH was significantly improved together with a reduction in MDA in a dose-dependent manner.

\section{Effect of LT on NF-KB and TLR-4}

The effect of $L T$ was further investigated on the expression of NF-KB and TLR-4 by western blot analysis. As shown in Fig 5 , the expression of NF-KB and TLR-4 were found highly elevated in the disease model group as compared to sham. The levels of these biomarkers were found significantly reduced in LT treated group in a dose-dependent manner as compared to the disease model group.

\section{Discussion}

Atopic dermatitis is a serious chronic inflammatory condition commonly occurring in children. It makes skin red and itchy which might be long-lasting. Till now AD has no concrete cure, yet medicines are available which only provide symptomatic treatments. Corticosteroids are the main component of the therapy against $A D$ together with anti-inflammatory agents. However, these medications due to serious side-effects could not be prescribed for long-term complications of AD (B. Brandt 2011; Ridao i Redondo 2012). Consequently, it is worthwhile to identify novel agents to treat AD multiple pathways with serious complications. Luteolin (LT), a flavonoid obtained from plants 

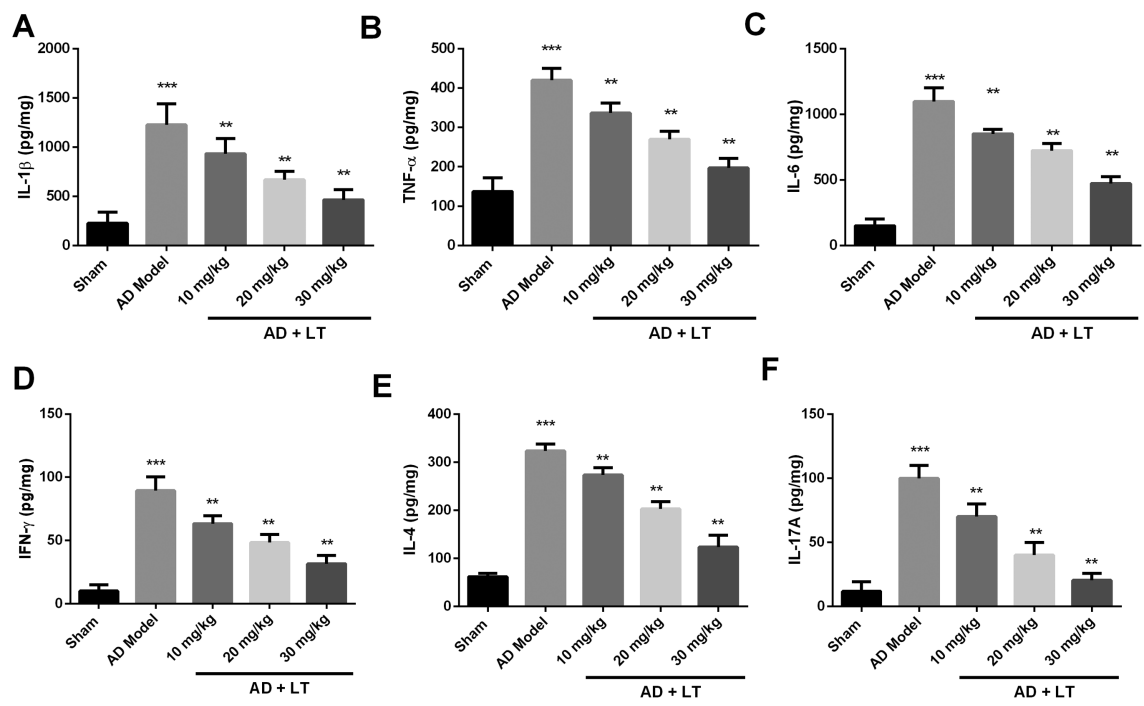

Figure 3. Effect of LT on pro-inflammatory cytokines, where, (A) IL-1 $\beta,(B)$ TNF- $\alpha,(C)$ IL-6, (D) IFN- $\gamma$, (E) IL-4, and (F) IL-17A. Values represent the mean \pm SEM. ${ }^{* * *} P<0.05$ vs control; ${ }^{* *} P<0.05$ VS $A D$, one-way analysis of variance (ANOVA) followed by Bonferroni post hoc test.
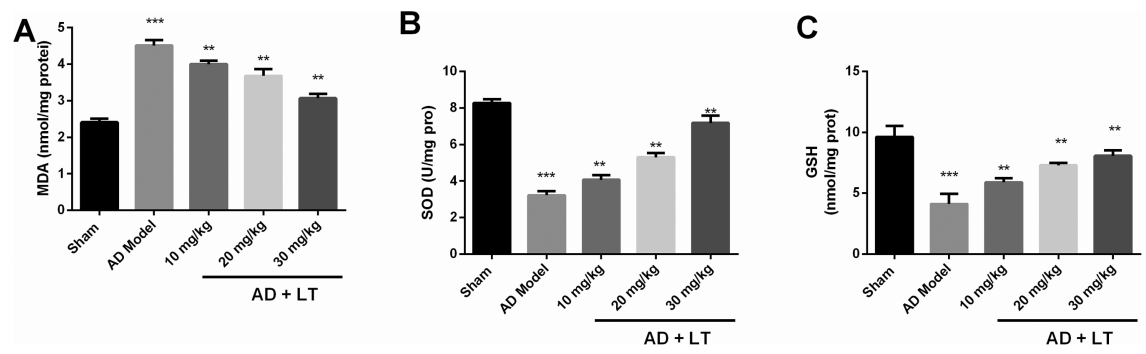

Figure 4. Effect of LT on the oxidative stress indices. Values represent the mean \pm SEM. ${ }^{* * *} P<0.05$ Vs control; ${ }^{* *} P<0.05$ Vs $A D$, one-way analysis of variance (ANOVA) followed by Bonferroni post hoc test.

A

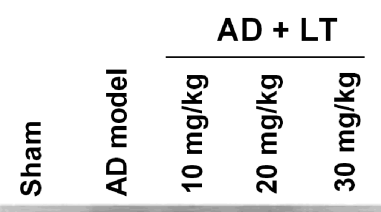

NF-KB

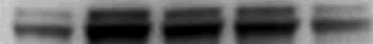

TLR-4

$\beta$-actin
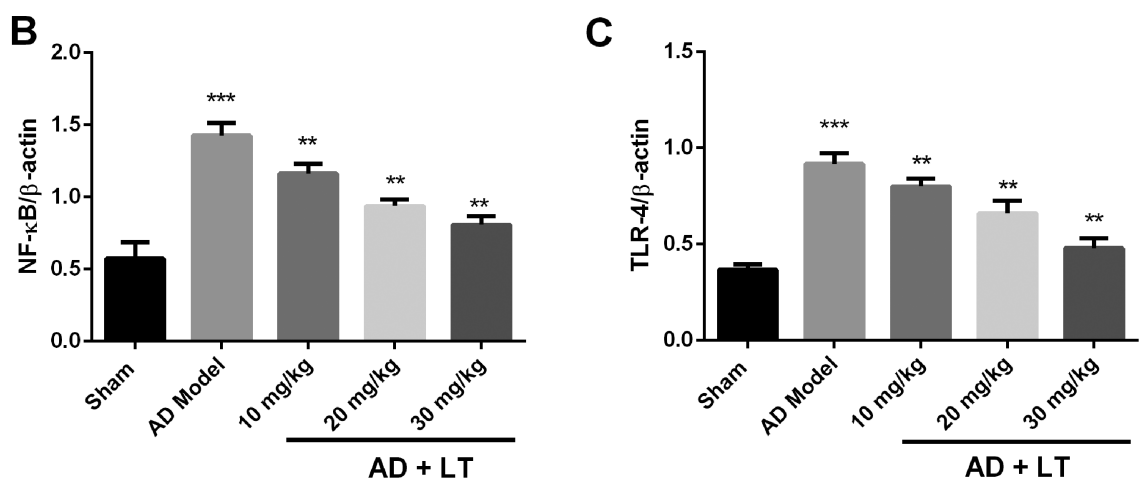

Figure 5. Effect of LT on NF-kB and TLR-4, where (A) western blot, and representative densitometry bar-graph showing the level of (B) NF-kB, and (C) TLR-4. Values represent the mean \pm SEM. ${ }^{* * *} P<0.05$ vs control; ${ }^{* *} P<0.05$ vs AD, one-way analysis of variance (ANOVA) followed by Bonferroni post hoc test. 
showed significant anti-oxidant and anti-inflammatory properties (Nabavi et al. 2015). Moreover, recently it also showed strong activity against $A D$ in canines. Thus, in the present study, for the first time, we have demonstrated the protective effect of LT in atopic dermatitis mediated via IgE and NF-kB. In the present study, we have utilized DNCB, which induces humanlike $A D$ symptoms in mice, such as, increased scratching behavior which induces skin thickening and damage to the skin barrier (Pickard et al. 2007; Newell et al. 2013). Various studies have demonstrated the elevated level of IgE in AD as a characteristic feature of the disease in response to various sensitizing agents, such as food, inhalants, microbes, etc. The high level of $\mathrm{IgE}$ is considered to provoke various IgE-mediated immune responses in AD. Therefore, initially, we examine the effect of $L T$ on the serum level of IgE after sensitization with DNCB in mice. It has been found that $L T$ causes a significant reduction in an elevated level of IgE suggesting its protective effect against LT via reducing IgE-mediated immune response (Kawakami et al. 2009; Liu et al. 2011; Horimukai et al. 2014). The beneficial effect of $L T$ was further confirmed on observing histopathology of the skin tissues, where LT significantly restored the normal architecture of skin tissue in a dose-dependent manner. Various laboratory and clinical findings have suggested the significance of elevated WBC cell population as an important biomarker after IgE-mediated immune response in AD (Salim et al. 2010; Gates and Atkinson 2012). Studies have also suggested that patients of $A D$ showed an increased level of eosinophils, thus, it is worthwhile to assess the effect of LT on the WBC cells population. It has been found that LT significantly reduces the level of these cells (neutrophils, basophils, eosinophils, monocytes, lymphocytes, and total WBC), possibly via reducing the elevated level of IgE. In $A D$, the higher expression of Th2-type cytokines (IL-4, IL-5, IL-13, and CCL18) linked with augmented cellular infiltration in the skin, the high circulating concentration of $\lg E$ and eosinophilia which is responsible for creating barrier defect in the skin. Moreover, the $\mathrm{TH}_{17}$-associated molecules (IL-17A, peptidase inhibitor 3/elafin, and CCL20) are found to always increased in both acute and chronic patients of AD. Studies have also shown the involvement of IL-17A in the immune dysregulation in patients with $A D$ by synergistically upregulating S100A7/8/9 together with IL-22 (B. Brandt 2011; Auriemma et al. 2013; Mu et al. 2014). Thus, considering the significant involvement of pro-inflammatory cytokines of (IL-1 $\beta$, IL-6, TNF- $\alpha$, IFN- $\gamma$, IL-4, and IL-17A) in the immune response of AD, next, we used ELISA assay to investigate the concentration of these cytokines in the blood serum after administration of LT. It has been found that LT causes a significant reduction of these cytokines which might be the reason for its anti-inflammatory effect against $A D$ in mice. Oxidative stress is another hallmark of $A D$ together with IgE-mediated immune response. Studies have shown a higher level of lipid peroxidation as evidenced by high serum MDA concentration in patients. Moreover, the dermal inflammation was often exacerbated in $A D$ together with higher oxidative stress due to reduced antioxidant capability (Okayama 2005; Ji and Li 2016). Thus, we have examined the level of LT on various indices of oxidative stress (MDA, SOD, and GSH). As shown in the present study, the LT treated group significantly restored the level of antioxidant ability as confirmed by an increased level of SOD and GSH together with the reduction in MDA in a dose-dependent manner. The increased oxidative stress is known to activate the NF-KB pathway to which is known to regulate various genes responsible for regulating inflammation and apoptosis (Nakamura et al. 2002; Matsushima et al. 2010; Sohn et al. 2011). During this activation, the NF-KB translocates from the cytoplasm to the nucleus to activate various genes. TLR4 is a transmembrane protein, a member of the toll-like receptor family, which belongs to the pattern recognition receptor (PRR) family. Various studies have shown that its activation induces intracellular signaling pathway NF-KB and inflammatory cytokine production which is responsible for activating the innate immune system in keratinocytes and compromise the epithelial defense barrier in AD (Bernard et al. 2012; Salimi et al. 2013; Biedermann et al. 2015). Keratinocytes express several innate immune receptors collectively referred to as pattern recognition receptors (PRRs) that enable them to respond to microbes or tissue damage by releasing a broad range of inflammatory mediators (eg, cytokines, chemokines, and AMPs). These PRRs can also affect tight junction integrity. For example, Tolllike receptor (TLR) agonists enhance the tightness of TJs and arguably limit penetration of surface proteins/ microbes. Therefore a defect in this innate immunemediated epidermal barrier repair process might lead to chronic inflammation from PRR signaling, as seen in patients with $A D$ (Kuo et al. 2013). Thus, in the last phase of the study, we aimed to investigate the effect 
of LT on the expression of NF-KB and TLR-4 via western blot (Havouis et al. 2000; Choi et al. 2017). It has been found that LT significantly reduces the expression of NF-KB and TLR-4. The results of our study were found in line with a study carried by Jo and colleagues where they have analyzed the effects of ethanol (EtOH) extract of the aerial parts of Stellera chamaejasme L. (Thymelaeaceae) on oxazolone or 2,4-dinitrochlorobenzene stimulated murine models of atopic dermatitis (Jo et al. 2019). Stellera chamaejasme contains diverse flavonoids and coumarins but the major active compound of the $\mathrm{EtOH}$ extract is luteolin 7-O-glucoside. They have shown that topical application of EtOH extract reduces serum level of IgE but also decreased epidermal thickening as well as transepidermal water loss and increased skin hydration in treated mice, pointing to a strong anti-atopic dermatitis activity. However, application of drug via topical route have own disadvantages, such as, the mice could lick the applied solution which may interfere with the results. Therefore, in our study we have administered LT via i.p. route.

\section{Conclusion}

Our study demonstrated the protective effect of Luteolin (LT) against atopic dermatitis via inhibition of lgE-mediated immune response via attenuation of NF$\mathrm{KB}$ activation. However, much detailed investigation is required to establish Luteolin (LT) as a clinical agent for atopic dermatitis.

\section{Animal welfare}

The present study followed international, national, and/or institutional guidelines for humane animal treatment and complied with relevant legislation.

\section{Declaration of conflicting interests}

The author(s) declared no potential conflicts of interest concerning the research, authorship, and/or publication of this article.

\section{Ethics approval}

Ethical approval to report this case was obtained from the institutional animal ethical committee of Central Hospital Affiliated to Shandong First Medical University, China for biomedical experiments.

\section{References}

Arikawa J, Ishibashi M, Kawashima M, et al (2002) Decreased levels of sphingosine, a natural antimicrobial agent, may be associated with vulnerability of the stratum corneum from patients with atopic dermatitis to colonization by staphylococcus aureus. J Invest Dermatol 119:433-439. doi: 10.1046/j.1523-1747.2002.01846.x.

Auriemma M, Vianale G, Amerio P, Reale M (2013) Cytokines and T cells in atopic dermatitis. Eur. Cytokine Netw. 24:37-44.

B. Brandt E (2011) Th2 Cytokines and Atopic Dermatitis. J Clin Cell Immunol 02: doi: 10.4172/2155-9899.1000110.

Bernard F-X, Morel F, Camus M, et al (2012) Keratinocytes under Fire of Proinflammatory Cytokines: Bona Fide Innate Immune Cells Involved in the Physiopathology of Chronic Atopic Dermatitis and Psoriasis. J Allergy 2012:1-10. doi: 10.1155/2012/718725.

Biedermann T, Skabytska Y, Kaesler S, Volz T (2015) Regulation of $T$ cell immunity in atopic dermatitis by microbes: The Yin and Yang of cutaneous inflammation. Front. Immunol. 6.

Choi JK, Jang YH, Lee S, et al (2017) Chrysin attenuates atopic dermatitis by suppressing inflammation of keratinocytes. Food Chem Toxicol 110:142-150. doi: 10.1016/j.fct.2017.10.025.

Emanuel S, Hawarden D (2018) Dermatitis. Curr Allergy Clin Immunol 31:164-165. doi: 10.5005/jp/books/14213_13.

Galli E, Cinicola B, Carello R, et al (2020) Atopic dermatitis. Acta Biomed. 91:1-10.

Gates MG, Atkinson TP (2012) Transient Hypereosinophilia and Markedly Elevated IgE as the Presenting Feature in DOCK8 (Dedicator of Cytokinesis 8) Deficiency. J Allergy Clin Immunol 129:AB159. doi: 10.1016/j.jaci.2011.12.356.

Gugliandolo E, Palma E, Cordaro M, et al (2020) Canine atopic dermatitis: Role of luteolin as new natural treatment. Vet Med Sci 6:926-932. doi: 10.1002/vms3.325.

Havouis S, Dumas G, Avé $P$, et al (2000) Mice lacking the transcription factor RelB develop T cell-dependent skin lesions similar to human atopic dermatitis. Eur J Immunol 30:23232332. doi: 10.1002/1521-4141(2000)30:8<2323::AIDIMMU2323>3.0.CO;2-H.

Horimukai K, Morita K, Narita M, et al (2014) Application of moisturizer to neonates prevents development of atopic dermatitis. J Allergy Clin Immunol 134:824-830.e6. doi: 10.1016/j. jaci.2014.07.060.

Ji H, Li XK (2016) Oxidative Stress in Atopic Dermatitis. Oxid. Med. Cell. Longev. 2016.

Jo BG, Park NJ, Jegal J, et al (2019) Stellera chamaejasme and Its Main Compound Luteolin 7- O -Glucoside Alleviates Skin Lesions in Oxazolone- and 2,4-DinitrochlorobenzeneStimulated Murine Models of Atopic Dermatitis. Planta Med 85:583-590. doi: 10.1055/a-0746-8698.

Kawakami T, Ando T, Kimura M, et al (2009) Mast cells in atopic dermatitis. Curr. Opin. Immunol. 21:666-678.

Kuo IH, Yoshida T, De Benedetto A, Beck LA (2013) The cutaneous innate immune response in patients with atopic dermatitis. J. Allergy Clin. Immunol. 131:266-278.

Langan SM, Irvine AD, Weidinger S (2020) Atopic dermatitis. Lancet 396:345-360. 
Leung DYM, Boguniewicz M, Howell MD, et al (2004) New insights into atopic dermatitis. J. Clin. Invest. 113:651-657.

Lin Y, Shi R, Wang X, Shen H-M (2008) Luteolin, a flavonoid with potential for cancer prevention and therapy. Curr Cancer Drug Targets 8:634-46. doi: 10.2174/156800908786241050.

Liu FT, Goodarzi H, Chen HY (2011) lgE, mast cells, and eosinophils in atopic dermatitis. Clin Rev Allergy Immunol 41:298310. doi: 10.1007/s12016-011-8252-4.

Marsella R, De Benedetto A (2017) Atopic dermatitis in animals and people: An update and comparative review. Vet. Sci. 4.

Matsushima Y, Kikkawa Y, Takada T, et al (2010) An Atopic Dermatitis-Like Skin Disease with Hyper-lgE-emia Develops in Mice Carrying a Spontaneous Recessive Point Mutation in the Traf3ip2 (Act1 / CIKS) Gene. J Immunol 185:2340-2349. doi: 10.4049/jimmunol.0900694.

Mu Z, Zhao Y, Liu X, et al (2014) Molecular Biology of Atopic Dermatitis. Clin. Rev. Allergy Immunol. 47:193-218.

Nabavi SF, Braidy N, Gortzi O, et al (2015) Luteolin as an anti-inflammatory and neuroprotective agent: A brief review. Brain Res. Bull. 119:1-11.

Nakamura H, Aoki M, Tamai K, et al (2002) Prevention and regression of atopic dermatitis by ointment containing NF-kB decoy oligodeoxynucleotides in NC/Nga atopic mouse model. Gene Ther 9:1221-1229. doi: 10.1038/sj.gt.3301724.

Newell L, Polak ME, Perera J, et al (2013) Sensitization via healthy skin programs Th2 responses in individuals with atopic dermatitis. J Invest Dermatol 133:2372-2380. doi: 10.1038/ jid.2013.148.
Okayama Y (2005) Oxidative stress in allergic and inflammatory skin diseases. Curr. Drug Targets Inflamm. Allergy 4:517-519.

Pickard C, Smith AM, Cooper H, et al (2007) Investigation of mechanisms underlying the T-cell response to the hapten 2,4-dinitrochlorobenzene. J Invest Dermatol 127:630-637. doi: $10.1038 /$ sj.jid.5700581.

Ridao i Redondo M (2012) Dermatitis atópica. Pediatr Integr 16:213-221. doi: 10.1016/s1761-2896(16)80892-4.

Salim N, Morra M, Nelson R (2010) Idiopathic CD4+ Lymphopenia Associated with a Heterozygous RAG1 Mutation in an Adult. Clin. Immunol. 135:S104-S105.

Salimi M, Barlow JL, Saunders SP, et al (2013) A role for IL-25 and IL-33-driven type-2 innate lymphoid cells in atopic dermatitis. J Exp Med 210:2939-2950. doi: 10.1084/jem.20130351.

Seelinger G, Merfort I, Schempp CM (2008) Anti-oxidant, antiinflammatory and anti-allergic activities of luteolin. Planta Med. 74:1667-1677.

Sohn EH, Jang SA, Joo H, et al (2011) Anti-allergic and anti-inflammatory effects of butanol extract from Arctium Lappa $\mathrm{L}$. Clin Mol Allergy 9:. doi: 10.1186/1476-7961-9-4.

Thomsen SF (2014) Atopic Dermatitis: Natural History, Diagnosis, and Treatment. ISRN Allergy 2014:1-7. doi: $10.1155 / 2014 / 354250$.

Wu PC, Chuo WH, Lin SC, et al (2019) Sclareol attenuates the development of atopic dermatitis induced by 2,4-dinitrochlorobenzene in mice. Immunopharmacol Immunotoxicol 41:109-116. doi: 10.1080/08923973.2018.1555846. 